\title{
Une langue en situation de handicap
}

\author{
Les sourds et la langue des signes face à la catégorie \\ du handicap
}

Pierre Schmitt

\begin{abstract}
La langue des signes est aujourd'hui reconnue en France au sein d'une loi dite sur le handicap. Victoire pour certains, impasse pour d'autres, la diversité de ses interprétations nous invite à réfléchir aux conditions de la catégorisation de la surdité comme handicap afin de mieux comprendre pourquoi les sourds la refusent, et pourquoi l'institutionnalisation de liens entre langue des signes et handicap est inquiétante. En premier lieu, à la recherche de discours et mobilisations ayant contribué à l'émergence d'une telle législation, nous prêterons attention au tournant initié par les « disability studies » puis à l'état des lieux de l'agenda politique du « réveil sourd ». Nous poursuivrons en exposant la stigmatisation de la langue des signes qui caractérise selon nous la loi, ce qui nous amènera à discuter la position du chercheur «militant» ainsi que les relations entre le politique, le social et la recherche.

Mots-clefs : handicap, déficience, catégorie, "disability studies », sourds, langue des signes.

In France, sign language is nowadays legitimated through a "disability law". Considered either as a victory or as a pitfall, its diverse interpretations invite us to question the categorization of deafness as a disability, so that we might achieve a better understanding of why deaf people reject it and why institutionalized links between sign language and disability are disturbing. Looking for discourses and social movements which have contributed to the emergence of such a law, we will first pay attention to the shifting point initiated by the disability studies and then to the progress of the political agenda of the "réveil sourd". We will then share our thoughts about the stigmatization of sign language implied by the law. This will lead us to discuss the position of the "militant" researcher and the relations between politics, society and research.
\end{abstract}

Keywords: disability, impairment, category, disability studies, deaf people, sign language

\section{Introduction}

Après une période d'interdiction de près de cent ans, la langue des signes est aujourd'hui reconnue officiellement en France au sein d'une loi dite sur le handicap. Cette loi 2005-102 «Pour l'égalité des droits et des chances, la participation et la 
citoyenneté des personnes handicapée » offre de nouvelles conditions à la mise en place de projets éducatifs bilingues langue des signes française/français et s'est donc rapidement rendue indispensable à l'action sur le terrain. Pourtant, une grande partie des sourds locuteurs de langues gestuelles ne se reconnaissent pas dans cette catégorie du handicap qui témoigne davantage du regard porté sur eux par la société que du discours qu'ils portent sur eux-mêmes.

C'est donc à partir de l'expérience des sourds que nous proposons ici une réflexion sur cette catégorie, sur les enjeux et modalités de la pérennisation législative de liens entre langue des signes et handicap. Autrement dit, à travers l'exploration des conséquences sociales de l'usage politique de la catégorisation de la surdité comme handicap, notamment en ce qui concerne l'éducation des jeunes sourds, il s'agira d'expliciter ce que les conditions de cette catégorisation font à la langue des signes et aux locuteurs de langues gestuelles, et ceci afin d'essayer de comprendre pourquoi les sourds la refusent, la contestent en partie et ce que cela nous dit sur la construction des catégories sociopolitiques.

Dans un premier temps, à partir de la synthèse de résultats d'enquêtes, nous exposerons la manière dont les sourds sont quelque peu noyés par le handicap. Dans un second temps, la réflexion proposée abordera la question du handicap en lien avec la reconnaissance officielle de la langue des signes en France, ce qui implique de revenir sur les mouvements sociaux qui ont permis à ces lois de voir le jour. Il s'agit d'une part des mouvements des personnes en situation de handicap en général, qui ont conduit à l'institutionnalisation de la catégorie du handicap, notamment à travers l'émergence d'un champ d'«études sur le handicap» ou disability studies, et d'autre part des mouvements propres aux sourds, mobilisés autour de la promotion de leur langue et de son statut ${ }^{1}$. Nous verrons ensuite comment, malgré la revendication d'un modèle social - respectivement du handicap et de la surdité - portée par ces mouvements, la langue des signes reste piégée par le stigmate de la déficience au sein de la loi de 2005. Enfin, entre compréhension et action, à travers un retour réflexif sur la diversité des acteurs et champs évoqués au fil de la discussion et leurs relations, nous reviendrons sur la manière dont la (dé)construction de la catégorie du handicap interroge la position du chercheur et surtout la pertinence de la séparation entre le politique, le social et la recherche.

1 Il ne s'agira pas tant ici de décrire en détail ces mouvements, puisqu'il existe des monographies abordant chacun de ces mouvements - y compris le mouvement des sourds (KERBOUC'H à paraitre; MiNGUY 2009) que de cerner les relations qu'ils entretiennent avec la reconnaissance officielle de la langue des signes. 


\section{Les sourds noyés par le handicap}

En ce qui concerne la présente réflexion, lorsque nous parlons de sourds, il s'agit de locuteurs de langues gestuelles, de ceux qui parlent la langue des signes, ceux que l'on appelle parfois les sourds signeurs, ceux que l'on appelait autrefois les sourds-muets, ceux qu'Yves Delaporte décrit comme se définissant eux-mêmes selon des critères linguistiques et culturels (DELAPORTE 2002). Ainsi, bien qu'il s'agisse d'individus que les normes médicales permettent de qualifier à des degrés divers de déficients auditifs, dans le cadre de mes travaux, être sourd ne signifie pas tant «ne pas entendre» que « parler avec ses mains ».

D'autre part, au regard de nos institutions, que l'on songe aux Maisons départementales des personnes handicapées $(\mathrm{MDPH})$ ou à la carte d'invalidité, les sourds relèvent de la catégorie des personnes en situation de handicap. Néanmoins, si les sourds s'accommodent des dispositifs de "compensation du handicap » permises par cette catégorisation, ils l'acceptent difficilement. Pour autant, à la fois catégorie de l'action publique, construction conceptuelle actualisée par différents courants de recherche en sciences sociales et représentation socialement dominante voire hégémonique pour appréhender la déficience sensorielle au sein de notre société, le handicap fait irrémédiablement partie du champ d'étude de la surdité, que l'on s'intéresse à lui comme outil d'analyse dont la pertinence est à évaluer ou à ce qu'il signifie, représente et implique dans les discours et pratiques des acteurs.

A cet égard, nous avons mené une enquête de terrain à partir de l'analyse de plusieurs corpus de photos et de documents variés abordant le handicap dans son ensemble. Cette enquête suggère clairement qu'il est difficile d'accéder à une autre représentation $\mathrm{du}$ handicap que celle du handicap moteur lorsque l'on consulte un support d'information qui traite du handicap de manière générale. D'autre part, dès que l'on emploie l'adjectif d'«handicapé» comme générique, ou que l'on représente iconographiquement la catégorie par un fauteuil roulant, chaque catégorie spécifique est noyée dans une indifférenciation dont le handicap moteur est le seul à émerger. Autrement dit, la catégorie française du handicap est fortement dominée, sémiotiquement et sémantiquement, par le handicap physique, alors que les sourds et les aveugles apparaissent comme une sous-minorité en retrait au sein de la minorité handicapée.

L'étude dont nous avons repris succinctement ici les conclusions les plus générales, souligne donc le fait que le handicap est une catégorie hétérogène, complexe, à utiliser avec précaution dans le cas des sourds, dans la mesure où il est aisé d'y relever le poids du handicap moteur, voire une certaine «domination symbolique du fauteuil roulant». Au delà d'un débat sur la réalité du handicap des sourds, ces observations permettent de fournir quelques données objectivées sur les raisons qui poussent les sourds à rejeter l'étiquette du handicap: comment leur reprocher de ne pas s'y reconnaître alors que la plupart du temps l'emploi de cette catégorie échoue à identifier les problématiques qui 
leur sont propres, qu'ils sont loin d'être les premiers à venir à l'esprit de ceux qui la verbalisent, et que leurs aspirations propres sont loin d'être au centre des politiques publiques qui s'y réfèrent?

\section{Handicap et déficience : (les limites de) l'apport des Disability Studies}

Après le constat de la faible représentation politique dont bénéficient les sourds au sein de la catégorie du handicap, il est nécessaire de revenir sur les disability studies et le transfert inachevé de leurs idées au reste de la société si l'on souhaite mieux comprendre les réactions ambivalentes suscitées par la reconnaissance de la langue des signes dans une loi sur le handicap. En effet, dans le monde anglo-saxon, l'institutionnalisation d'équipes et de départements spécialisés dans les «études sur le handicap » constitue l'une des expressions des mouvements sociaux des personnes en situation de handicap - principalement physique - initiés aux Etats-Unis et qui influencèrent le contenu et la forme des législations contemporaines en matière de handicap. À l'instar de Lennard Davis (2007), on peut peut-être regretter que les sourds ne rejoignent pas ces mouvements de personnes handicapées qui revendiquent par exemple l'idée de « differently able » au lieu de celle de « disabled », celle d'autrement ou «différemment capable» au lieu de celle d' «incapable», ce concept rejoignant largement la mise en avant par les sourds de leurs capacités autres - notamment la pratique d'une langue autre ou l'établissement d'un rapport au monde principalement visuel plutôt qu'auditif - au lieu d'incapacités. Cependant, leur position, leur rejet du handicap nous en dit beaucoup sur la difficile transmission de ces idées à l'ensemble de la société : alors que les disability studies ont développé et tenté de diffuser un modèle social du handicap reposant largement sur la distinction entre handicap et déficience, les sourds refusent néanmoins le handicap car ils constatent quotidiennement qu'il s'agit d'un avatar du stigmate de la déficience.

Ainsi, si les sourds refusent leur catégorisation sociale en tant que « handicapés », c'est principalement parce que tout en proposant une lecture de la surdité comme différence culturelle et linguistique, ils sont conscients de l'inadéquation entre cette lecture et les représentations communes du handicap - que nombre d'entre eux partagent d'ailleurs : malgré l'adoption d'un nouveau vocable, telle que l'expression «personne en situation de handicap », émergeant afin de contextualiser et socialiser les difficultés rencontrées par une personne porteuse d'une déficience en les plaçant à un niveau interactionnel et collectif, et non plus individuel et permanent, la plupart des acteurs politiques et sociaux ne se sont pas appropriés les subtilités introduites par les évolutions conceptuelles issues des disability studies et perçoivent la situation de handicap comme l'attribut permanent d'une personne déficiente, diminuée. L'étiquette a changé, mais le contenu demeure largement identique : une personne en situation de handicap, c'est un infirme. D'ailleurs, malgré un certain nombre de changements de terminologie, il est 
probablement signifiant que les sigles GIG - Grand infirme de guerre - et GIC Grand infirme civil - soient toujours d'actualité dans la signalétique de l'espace public pour désigner les places de parking réservées aux "personnes en situation de handicap ». Or les sourds ne se perçoivent pas comme des infirmes, mais comme des individus différents dont le handicap est avant tout communicationnel, fruit d'une interaction, et donc partagé.

Ces remarques permettent de qualifier le constat du sociologue d'euphémisme tant les représentations sociales actuelles du handicap peuvent paraittre éloignées de sa définition par les sciences sociales contemporaines : « la perspective qui tend à associer le handicap à une interaction entre l'individu, les contraintes qu'impose sa particularité et son environnement et non à une caractéristique intrinsèque de l'individu, n'est pas encore complètement admise » (EBERSOLD 2003, pp.32-33).

Le modèle «social », ou culturel du handicap proposé par les disability studies a tout de même gagné du terrain puisqu'on le retrouve en partie au sein des lois, notamment françaises et américaines, qui assurent la «gestion » politique du handicap. Pour autant, face à l'inertie du modèle médical, tout dépend de l'interprétation de ces lois et de la capacité des mouvements qui les ont portés à mettre en œuvre leur application dans les conditions sociales désirées. Linda Krieger indique ainsi que les lois ne peuvent changer seules la société et souligne la nécessité d'une «stratégie de changement social, idéologique et institutionnel » à long terme, dépassant la seule adoption de lois, pour les mouvements qui souhaitent "changer les normes sociales et les pratiques institutionnelles établies » (KRIEGER 2008).

En France, au delà des mouvements propres aux personnes sourdes revendiquant leur émancipation, la reconnaissance de la langue des signes au sein de la loi de 2005 doit donc être mise en relation avec l'émergence de législations sur le handicap relevant en partie du modèle social du handicap développé par les disability studies. Ces remarques permettent de faire apparaître une double culturalisation des représentations liées au handicap: culturalisation de la définition du handicap à partir de l'émergence d'un modèle social, et culturalisation du processus de création, de négociation et de transformation de la catégorie sociopolitique du handicap en soulignant qu'elle est le fruit de mouvements sociaux, de la mobilisation d'acteurs qui produisent des discours alternatifs dans un contexte historique et culturel donné, avant que ceux-ci ne soient relayés et pérennisés politiquement au sein de lois en faveur des personnes en situation de handicap qui déterminent les conditions de l'action publique. La prise en compte de la dimension culturelle de ce processus de création d'une catégorie particulière peut assurément être étendue à n'importe quelle catégorie sociopolitique, qui indépendamment du degré d'objectivation revendiquée par son élévation au rang de catégorie de l'action publique, est initialement construite et élaborée par d'autres champs du social que celui du politique et du législatif. Autrement dit, ces lois sont l'expression de représentations sociales et culturelles, qu'elles soient communes ou issues de mouvements sociaux spécifiques. À ce titre, l'étiquette de «socio-politique» 
témoigne de ces relations solidaires entre le social et le politique, entre les mouvements sociaux et le changement au sein des institutions publiques.

\section{Du mouvement des sourds aux impasses de la reconnaissance de la langue des signes}

En dépit des tensions entre modèle social et modèle médical, handicap et déficience, représentations communes et discours des disability studies, la loi sur le handicap a donc fourni un cadre à la reconnaissance de la langue des signes en France. Cependant, cette reconnaissance constitue l'aboutissement de plus de trente ans de luttes sociales centrées essentiellement sur des questions de langue et d'éducation. Émergeant dans les années soixante-dix, ce mouvement est couramment nommé « réveil sourd », reprenant une expression idiomatique propre à la langue des signes française. Cependant, une attention particulière à la diversité des acteurs alors mobilisés, de même qu'aux chevaux de bataille retenus par le mouvement afin d'entériner une reconnaissance effective de la langue des signes, rend évident que si l'émancipation des sourds fut bien l'objectif du «réveil sourd» la réalisation de cet objectif détermina un agenda politique où l'établissement de rapports égalitaires et solidaires entre sourds et entendants intervenait à la fois comme point de départ et point d'arrivée de toute action à entreprendre, et impliquait donc une mobilisation commune, complémentaire, de la part des sourds et des entendants.

Ainsi, il est notamment possible d'insister pour le cas français sur une certaine dimension académique du « réveil sourd ». Il ne s'agit pas de voler la vedette aux sourds. Bien au contraire, il s'agit de souligner qu'en France, l'ouverture des sourds $a u(x)$ monde(s) scientifique(s) et entendant, par la collaboration avec des chercheurs, a dès le départ été un des éléments caractéristiques de leur mobilisation politique. D'ailleurs, il est important de garder à l'esprit que si la réduction de la fracture entre monde sourd et monde académique constitua à la fois une expression et une condition du «réveil sourd ", elle intervient précisément par le renouveau de relations privilégiées avec les Etats-Unis et leur contexte particulier. En effet, alors que le sociologue Bernard Mottez importe les travaux du linguiste William Stokoe qui avait établi dans les années cinquante et soixante le statut linguistique de la langue des signes américaine, des «stages» furent organisés à l'intention de sourds adultes et de parents entendants d'enfants sourds, au sein de l'université de Gallaudet -établissement d'enseignement supérieur où la langue des signes est langue d'enseignement aux côtés de l'anglais, où sourds et entendants partagent chairs et laboratoires - où résidait l'équipe de recherche de Stokoe. Interpréter le milieu académique comme un acteur « entendant» n'est donc possible que par une analyse tronquée qui ne tient pas compte de la dimension transnationale du « réveil sourd ». À l'inverse, comme nous allons le voir à présent, il est tout aussi erroné d'identifier les avancées de terrain du mouvement comme relevant uniquement d'acteurs «sourds ». 
Rapidement, il fut établi que les efforts devaient être concentrés vers trois initiatives majeures : la mise en place de lieux d'apprentissage pour tous ceux qui manifesteraient le besoin ou simplement l'envie d'apprendre la langue des signes, la structuration d'un corps professionnel d'interprètes, et le retour de la langue des signes comme langue d'enseignement dans l'éducation des enfants sourds (MOTTEZ \& MARKOWICZ 1980).

Dans l'article de 1980 analysant le mouvement social en marche pour la reconnaissance de la langue des signes française, Bernard Mottez et Harry Markowicz se montraient optimistes quant aux deux premiers points, étant donné les progrès réalisés dans ces domaines depuis 1975, tels que la création du théâtre de Vincennes l'actuel International Visual Theater (IVT) - ou de l'Association nationale française des interprètes pour déficients auditifs - ANFIDA, ancêtre de l'actuelle AFILS, Association française des interprètes en langues des signes ${ }^{2}$. Cependant, l'inertie du milieu scolaire, voire l'hostilité des institutions vis-à-vis de réformes accordant à la langue des signes la place que les mesures faisant suite au congrès de Milan de 1880 avaient achevé de lui confisquer, inquiétaient davantage.

Presque trente ans plus tard, force est de constater l'exactitude du tableau dressé. Aujourd'hui, si les besoins en interprètes ne sont toujours pas couverts, que ces derniers sont amenés à militer quotidiennement afin que leurs conditions de travail respectent le cadre déontologique de leur profession, il convient de souligner que la question de l'interprétation a tout de même évolué qualitativement sur le territoire français ${ }^{3}$. De même, au delà de leurs domaines de compétences variées, un grand nombre de structures socioculturelles proposent actuellement des cours de langue des signes, du prestigieux IVT aux associations locales de sourds dans un nombre important de villes de taille moyenne, alors qu'à l'échelle nationale, l'enseignement de la langue des signes se dote peu à peu d' "un cadre analogue à celui des autres langues [...] (référentiel, document pédagogique, diplôme de compétence en langues, etc) » (DALLE 2003, p.57).

Parallèlement à cette progression de la place de la langue des signes dont témoignent le développement de l'interprétation professionnelle et son enseignement auprès d'un nombre toujours croissant d'entendants, l'éducation des enfants sourds constitue donc la véritable ombre au tableau du «réveil sourd». Alors que la loi de 2005 prétend garantir le droit à une éducation bilingue, la plupart des acteurs du milieu reconnaissent

\footnotetext{
2 Pour une histoire de l'interprétation en langue des signes en France depuis les années 1970, voir l'article de Christine Quipourt et Patrick Gache publié dans Langue française (QUIPOURT \& GACHE 2003).

3 «Il va de soi que le nombre d'interprètes en France est nettement insuffisant (pour rappel, environ 120 interprètes répartis de manière hétérogène sur le territoire) pour répondre à la demande » (QUIPOURT \& GACHE 2003, p.109). «A titre de comparaison, les Etats-Unis comptent à peu près 5000 interprètes en ASL, et il y a plus d'interprètes en langue des signes dans la seule ville de Stockholm que sur tout le territoire français » (ibid.).
} 
que moins de cinq écoles sur tout le territoire peuvent prétendre fournir un tel parcours. À l'échelle de la demande, alors que le déménagement ou la mobilisation locale sont les seules alternatives, la situation s'apparente donc à un " néant en matière de classes bilingues »(FrUCHARD 2007, p.16).

\section{Le stigmate de la déficience}

Ni la loi de 2005, ni le récent plan en faveur des personnes en situation de handicap n'ont permis de changer significativement cette situation. À propos de ce dernier, dans un communiqué diffusé sur différents forums et sites internet, André Meynard parle d' « une entreprise de 'communication' déconnectée des problèmes du terrain», assurant que «les bonnes intentions affichées [...] ne prennent aucunement en compte les mesures préventives dégagées par les cliniciens travaillant dans ce domaine depuis de longues années ». Dans la lignée d'une critique de la loi de 2005 et du contexte général de prise en charge des enfants sourds, il dénonce «la mise en place d'une filière de soin [...] [qui] demeure à ce jour hautement préoccupante précisément car elle porte atteinte à l'accès précoce à ces réseaux langagiers [ceux de la langue des signes] pour ces sujets et leurs familles ».

Concrètement, bien que la loi reconnaisse la langue des signes comme une langue à part entière, les parents sont essentiellement pris en charge par un milieu médical globalement hostile aux langues des signes, ce qui permet d'être critique quant aux conditions de mise en œuvre d'un «libre choix» de l'éducation bilingue. Surtout, il s'avère inquiétant que la loi entérine le pendant inverse de ce choix : la liberté de refuser à leur enfant tout accès aux langues gestuelles, indépendamment de son degré de surdité, pour des parents entendants principalement orientés par les représentations dominantes de la surdité comme handicap du langage et de la parole. Dans cette perspective de «libre refus » de la langue des signes, désormais légitimé par un cadre législatif renforcé, l'enfant est principalement perçu comme déficient et le recours à la langue des signes comme l'expression de l'échec de la réparation.

Pourtant, à propos des étudiants handicapés, Serge Ebersold souligne à quel point «tout raisonnement en termes d'incapacité ou de déficience constitue un frein majeur» à leur accueil puisqu'il déplace l'attention qui devrait être portée aux aménagements envisageables vers les impossibilités de l'étudiant (EBERSOLD 2003, pp.32-33).

Ces remarques semblent tout aussi pertinentes à l'égard de la scolarisation des enfants sourds tandis que l'acceptation de la langue des signes comme langue à part entière, langue enseignée et langue d'enseignement qui ne met pas en danger l'acquisition de compétences en français, à l'écrit ou à l'oral, relève des aménagements permettant de concilier les exigences scolaires et la présence d'enfants présentant une déficience auditive empêchant l'acquisition du français de manière «naturelle» au sein de la famille, c'est-à-dire des enfants dont la maittrise orale du français est insatisfaisante pour 
les échanges scolaires, et dont les conditions d'accès au français écrit - avec ou sans langue des signes - ne seront jamais identiques à celles d'enfants entendants 4 .

Les impasses de la reconnaissance concrète et effective de la langue des signes et des bénéfices qu'elle peut apporter à tous les enfants sourds, indépendamment de leur degré de surdité, sont à mettre en relation directe avec la stricte catégorisation de la surdité comme handicap dans un contexte où le modèle médical domine encore les représentations. Dans ce contexte où handicap et déficience ne sont pas clairement distingués, comment serait-il possible d'obtenir une reconnaissance pleine et entière de la langue des signes au sein d'une loi sur le handicap? Comment celle-ci pourrait-elle garantir de véritables aménagements permettant de couvrir et de susciter la demande en matière d'éducation bilingue? A divers égards, perçue comme un pis-aller pour les enfants sourds pour qui l'éducation ordinaire est compromise, il apparait que par ricochet des représentations communes de la surdité et de l'échec de la diffusion du modèle social du handicap, la langue des signes est paradoxalement enfermée dans l'image de langue de la déficience par sa reconnaissance même au sein d'une loi sur le handicap. Ainsi, de la même manière que les situations de handicap liées à la surdité sont renvoyées au rang de caractéristiques intrinsèques de l'individu, la langue des signes n'est pas distinguée de la déficience elle-même. Elle n'est pas perçue comme une production culturelle collective à même de résorber le handicap en plaçant l'enfant sourd dans des conditions d'égalité d'être parlant - et d'apprenant dans le cas des écoles bilingues - mais bien plus comme une expression de la déficience dont elle partage le stigmate.

Alors que le refus des langues gestuelles relevait jusque là d'une situation de fait, reflet des pratiques et discours encadrant la surdité au sein de notre société, il constitue désormais un droit, soutenu juridiquement par une loi sur le handicap qui relève davantage du modèle médical que du modèle social du handicap en ce qui concerne la surdité. Dans la perspective de la promotion de l'accès des enfants sourds aux langues des signes, position aujourd'hui défendue par de nombreux acteurs de terrain, sourds et endendants, ainsi que par tous les travaux contemporains de sciences humaines et sociales - linguistique, sociologie, anthropologie sociale, pédagogie, didactique des langues -, ne s'agirait-il pas davantage d'un danger que d'une victoire?

${ }^{4}$ Andrea Benvenuto insiste sur le fait que la langue vocale «ne peut être acquise par les enfants [sourds] de manière naturelle sans une intelligence autre, celle de l'orthophoniste » (BENVENUTO 2008, p.6) 


\section{Le politique, le social et la recherche}

Et si certains entendants et sourds se mobilisent pour la langue des signes, valorisent les aspects culturels de la surdité, il n'y a pas de retournement de stigmate dans ce qui n'est finalement que la revendication de la fin de l'hypocrisie ou de la schizophrénie ambiante - à chacun de déterminer la sincérité des acteurs - : si les langues gestuelles sont des langues au même titre que les langues vocales, que les sourds sont fondamentalement des individus complets au même titre que les entendants, néanmoins ponctuellement placés en situation de handicap, alors l'éducation bilingue devrait être le minimum accordé à tous les enfants sourds dont la déficience auditive rend difficile et complexe l'accès à la maîtrise orale des langues vocales ${ }^{5}$. Ce n'est pas sortir de la réserve du chercheur que de permettre au lecteur de mesurer lui-même l'écart entre le discours et les pratiques des institutions politiques et sociales françaises contemporaines, que de souligner les contradictions dans leur attitude face aux langues gestuelles, que d'accorder aux défenseurs de ces dernières le crédit de leurs arguments quand les sciences humaines et sociales contredisent chaque jour davantage les positions hostiles à la langue des signes. Alors qu'à travers la reconnaissance officielle de la langue des signes et le droit à l'éducation bilingue, les textes de lois et discours politiques suggèrent que le sourd est accepté dans sa différence linguistique et culturelle, dans le contexte actuel, «les parents entendants d'enfant sourd n'ont en définitive aucun choix» (FRUCHARD 2007, p.1) en matière d'éducation.

A la lumière des recherches contemporaines, bien que toujours minoritaire, et souvent perçu comme militant, déconstruisant en partie la catégorie sociopolitique du handicap, c'est aujourd'hui le discours des sourds qui semble le plus proche des positions objectivées des sciences humaines et sociales. Ainsi, au delà de domaines d'expertise variés, d'outils disciplinaires divers, comment se fait-il qu'un si grand consensus puisse exister parmi les chercheurs pour accorder à la langue des signes le statut de langue d'intégration des sourds (BENVENUto 2008; DeLaporTe 2002; Duranti 1997; LACHANCE 2007; LE BRETON 2006; SACKS 1989; SEGUILLON 1998)? Est-il désormais encore possible d'accorder un statut scientifique aux positions contraires dont on perçoit avec de plus en plus de difficulté les fondements empiriques, mais dont les relations avec un modèle biomédical du handicap sont évidentes?

\footnotetext{
5 Yves Delaporte invite à une réflexion similaire: "si la langue des signes est une langue authentique, si elle permet de tout dire et de tout traduire, si elle possède les mêmes richesses lexicales et syntaxiques que toutes les autres langues humaines, alors il faut bien admettre que les sourds ont raison sur toute la ligne dans leur revendication d'un enseignement au moyen de cette langue, et quand ils prétendent qu'ils sont tout autre chose que des malades » (DELAPORTE 2002, p.9)
} 
Combien de temps faudra-t-il encore souffrir que les travaux de spécialistes puissent être qualifiés de "partisans » ou de " militants » par ceux qui n'ont de connaissance des sourds que leurs présupposés hostiles à la langue des signes? Tout comme «les interprètes ne sont pas les représentants des sourds, mais la façon dont la société considérera leur métier est complètement dépendante du regard politique porté sur les sourds » (QUIPOURT \& GACHE 2003, p.109), le jugement de valeur porté sur les travaux qui s'éloignent par leurs résultats des positions médicales stigmatisant la langue des signes, qui se rapprochent de façon mécanique du discours diamétralement opposé des sourds, reflète non pas un contre-savoir académique qui leur serait opposé mais bel et bien une méconnaissance des situations concrètes vécues par les sourds. En effet, le miroir de la spécialisation des représentations issues de l'expérience des sourds, de son partage et de son étude, c'est l'ignorance de cette expérience caractérisant les représentations strictement déficitaires. Autrement dit, tel un écho du modèle social du handicap, les sourds détiennent une part de vérité lorsqu'ils « attribuent leurs difficultés, non à leur surdité, mais au fait que les entendants sont incapables de comprendre ce que c'est que d'être sourd» (DELAPORTE 2002, p.13).

Ecartant la chimère d'une neutralité impossible à poursuivre lorsque l'on considère que la recherche, de même que le politique, ne sont que des espaces particuliers de ce social sur lequel ils tiennent un discours ou tentent d'agir, le développement d'une réflexion sur la construction des catégories sociopolitiques menée à partir des conditions de la reconnaissance de la langue des signes au sein de lois sur le handicap nous invite à considérer un rapport politique à nos disciplines et au politique.

La question de l'objectivation propre à la production de catégories pouvant être reprises par le politique dans la poursuite de l'action publique permet surtout de souligner les transferts inachevés de concepts et de modèles, à l'instar du modèle social du handicap. Ce qui semble pouvoir être le mieux objectivé, c'est précisément le manque d'objectivation à l'œuvre dans la reprise de ces catégories et l'effet de phagocytage par les représentations dominantes, ici, celles qui font du handicap une caractéristique intrinsèque de l'individu et enferment la langue des signes dans le statut de langue de la déficience dans le mouvement même de sa reconnaissance officielle.

La construction et la déconstruction de catégories sociopolitiques apparaissent également comme un processus où la recherche en sciences sociales et les mouvements sociaux ne sont guère distinguables, qu'il s'agisse du «réveil sourd» ou de l'institutionnalisation des disability studies. La compréhension et l'action apparaissent donc comme solidaires. Entre le politique et la recherche, il pourrait cependant exister une proportion inverse entre les moments d'usage des catégories, et les moments de construction et de déconstruction. En effet, l'apport principal du postmodernisme, c'est peut-être l'invitation pour le chercheur à avouer qu'il ne sait pas, ou en tout cas qu'il ne sait pas tout, qu'il cherche. Selon nous, la stabilité des catégories qu'il emploie ne devrait pas avoir d'autre fin que la suspension momentanée de l'analyse afin de transmettre un savoir au moment de l'écriture, alors que celle-ci permet également d'expliciter les 
doutes et imperfections relatifs aux catégories proposées. En ce qui concerne le politique, de même que " pour l'anthropologie dominante, les Indiens existent puisque l'anthropologie les a rencontrés» (AMSELLE 2010), l'action publique suggère que les catégories qu'elle met en pratique existent. Alors que certains acteurs du champ politique manquent certainement de recul vis-à-vis des catégories auxquels ils ont recours, il est dans tous les cas complexe pour eux de ne pas afficher publiquement une certaine suspension de jugement lorsqu'ils font face à leur électorat. Ainsi, le temps de la consultation est donc largement réduit à la phase d'élaboration des lois, alors que leur application témoigne d'une sorte d'amnésie, présentant les catégories comme objectives, renvoyant ceux qui continuent de les déconstruire et de les contester au rang de militants, quand bien même la consultation suivante acceptera éventuellement leur revendications.

Alors que pour déconstruire cet indien que l'anthropologue a rencontré, Jean-Loup Amselle nous invite à interroger « le mode d'existence de ces catégories ethniques, leur mode d'apparition, leur historicité, leur champ sémantique, leur performativité, etc. » (AMSELLE 2010), je reprendrai cette invitation dans le cadre de l'analyse des catégories sociopolitiques telles que le handicap, dans la mesure où l'adoption d'un modèle médical ou social dans l'appréhension de la surdité détermine largement le statut accordé à la langue des signes, influence le destin des enfants sourds.

Dans le contexte contemporain français, la reconnaissance de la langue des signes ne représente dans tous les cas qu'un volet de la principale loi assurant à la catégorie sociopolitique du handicap ses contours. Au delà de l'hétérogénéité et des déséquilibres de cette catégorie, nous avons voulu souligner l'incomplète diffusion du modèle social du handicap proposé par les «études sur le handicap » ainsi que l'inertie du modèle médical et des institutions politiques et sociales dont témoigne le fond et la forme de la loi, son interprétation et son application. Il en résulte une persistance de la stigmatisation de la langue des signes, concrétisée par un «néant en matière de classes bilingues » et l'implicite légitimation d'un « libre refus » de la langue des signes dans un contexte qui lui est hostile. Enfin, refusant l'étiquette de militant pour ces observations et conclusions que nous jugeons objectivées, nous avons souhaité interroger les relations entre le politique, le social et la recherche, proposant en quelque sorte une mise en abîme des enjeux que cet article, notre position et notre démarche, partagent avec l'histoire et le rôle du « réveil sourd » et des disability studies.

Et si pour conclure, nous affirmerons une dernière fois que la langue des signes n'est pas déficiente, force est de constater qu'elle est bel et bien en situation de handicap, puisqu'elle est stigmatisée, minorée socialement et politiquement. En dépit de sa reconnaissance officielle et de l'instauration d'un droit à l'éducation bilingue, « elle n'est encore qu'exceptionnellement langue enseignée dans les établissements accueillant des jeunes sourds et, plus rarement encore, langue d'enseignement » (GARCIA \& DERYCKE 2010, p.7). Alors que la langue des signes demeure attachée au corps du sourd, perçue comme un reflet de sa déficience, sa reconnaissance pleine et entière ne pourra 
intervenir qu'à travers une déterritorialisation singulière: on ne pourra envisager garantir l'égalité des droits et des chances, la participation et la citoyenneté des sourds que lorsque leur langue cessera d'être handicapée par le stigmate de la déficience dont ils sont porteurs, pour devenir langue parmi les langues, une langue à personne, librement accessible à tous... les enfants sourds.

\section{Bibliographie}

AMSELLE J.-L. (2010): « Le retour de l'indigène ». In L'Homme, n¹94, pp.131-138.

Benvenuto A. (2008): «De l'égalité des intelligences à l'anti-pédagogie de la surdité ». In Les carnets de La Persagotière, $\mathrm{n}^{\circ} 7$.

DALLE P. (2003): «La place de la langue des signes dans le milieu institutionnel de l'éducation: enjeux, blocages et évolutions ». In Langue française, n¹37, pp.32-59.

DAVIS L. (2007): «Deafness and the Riddle of Identity ». In The Chronicle of Higher Education, 12 janvier 2007.

Delaporte Y. (2002): Les sourds, c'est comme ça. Paris: Editions de la Maison des sciences de l'homme.

Duranti A. (1997): Linguistic Anthropology. Cambridge: Cambridge University Press.

EBERSOLD S., EvANS P. (2003): Les étudiants handicapés dans l'enseignement supérieur. Paris: OCDE.

FRUCHARD M-L. (2007): «Parents entendants, les raisons d'un non choix pour leur enfant sourd ». Intervention dans le cadre de la journée d'étude du GERS, Le jeune enfant sourd: consensus et controverses, novembre 2007.

GARCIA B., DERYCKE M. (2010): «Introduction ». In Langage \& société, n¹31, pp.5-17.

KRIEGER L. (2008): «Un traitement égal Versus un traitement en tant qu'égal», Intervention dans le cadre de la journée d'étude du programme de l'EHESS Handicap et Sciences sociales, juin 2008.

Kerbouc'H S. (à paraitre): Le Réveil Sourd (1971 - 2006). Thèse de sociologie. Paris: EHESS.

LE BRETON D. (2006): La saveur du monde. Un anthropologie des sens. Paris: Editions Métailié.

MEYNARD A. (1995): Quand les mains prennent la parole. Ramonville Saint-Agne: Erès.

Minguy A. (2009): Le réveil Sourd en France. Pour une perspective bilingue. Paris: L'Harmattan. 
MotTez B., Markowicz H. (1980): «The Social Movement Surrounding French Sign Language ", in BAKER C., BATTISON R., ed. (1980): Sign Language and The Deaf Community. Essays in Honor of William C. Stokoe. Silver Spring: Publications of the National Association of the Deaf.

MOтTEZ B. (2006): Les Sourds existent-ils ?. Paris: L'Harmattan.

QUIPOURT C., GACHE P. (2003): «Interpréter en langue des signes : un acte militant ? ». In Langue française, $\mathrm{n}^{\circ} 137$, pp.105-113.

SACKS O. (1989): Seeing Voices. A Journey Into the World of the Deaf. Berkeley: University of California Press.

SEGUILLON D. (1998): De la gymnastique amorosienne au sport silencieux: le corps du jeune sourd entre orthopédie et intégration ou l'histoire d'une éducation à Corps et à cri. 1822-1937. Thèse de doctorat en STAPS. Bordeaux: Université Vi. 